Politics: A Sociological Perspective". Against the background of the deepening climate crisis, Spaargaren argued that the empowerment of private consumers as co-makers of change is an issue which deserves attention both from a theoretical and policy making perspective. Using a practice-oriented perspective of the role of human agents in climate change helps to emphasize agency in environmental change without lapsing into individualistic models of change, he argued.

Following the tradition started in Lüneburg in 2007, a workshop was also launched at this year's meeting. To stimulate a lively discussion, a background paper, co-authored by Huib de Vriend and Anna Wesselink, was circulated before the conference. Huib de Vriend, an engineer from the Technical University of Delft and director of the EcoShape Programme of the Netherlands not only attended his very first sociology meeting that day but with his co-author Anna Wesselink (University of Leeds, UK) he critically presented the EcoShape Programme with a talk on "Building with Nature: Ecodynamic Design in Practice". The goal of the Dutch programme is to design and shape the Dutch coast line by using dunes and beaches together with elements such as rocks and jetties with novel technologies. The invited speakers Gert Spaargaren (Wageningen) and Wolfgang Krohn (Bielefeld University, Germany) agreed with the audience that the idea of "Building with Nature" can be seen as a good example to foster win-win solutions for society and nature. On the other hand they also pointed to the many obstacles and unintended side effects that large landscape design processes based on novel approaches in hydraulic engineering and their relationship to the ecosystem dynamics can bring.

The closing plenary speech was delivered by Hellmuth Lange (University of Bremen, Germany), who discussed "First and Third World Environmentalism: Competing Concepts or Two Sides of the Same Coin?" Lange unfolded in detail what First and Third World environmentalism can mean from different disciplinary perspectives. He also discussed on how much globalization leads to a blurring of any clear boundaries between both "environmentalisms". Based on this debate, Lange ended by discussing a list of research themes which will certainly gain im- portance for environmental sociology in the near future, fostered by the globalization of (un-)sustainable lifestyles, consumption patterns, as well as environmental awareness and behaviour. In short, there is more work for environmental sociologists to be done than ever before.

With regard to the content of the overall conference theme, the meeting has supported the view that European environmental sociology is increasingly forging links with other disciplines, thus highlighting the inter- and even transdisciplinary potential of sociology as well as - at least on the local level - its strong focus on pragmatic solutions of environmental problems. Although the venue for the 3rd German Environmental Sociology Summit has not been finalized, there is good reason to look forward to the next meeting in November 2011.

$\langle\gg$

\section{Noch große Defizite im Management von Abfällen} Tagungsbericht vom „II. Simposio Iberoamericano de Ingeniera de Residuos"

\section{Barranquilla, Kolumbien, 24.-25. September 2009}

\section{von Klaus-Rainer Bräutigam, ITAS}

Das „Nachhaltige Management von Abfällen” war das Thema des Zweiten Iberoamerikanischen Symposiums zum Management von Abfällen, das am 24. und 25. September an der Universidad del Norte in Barranquilla (Kolumbien) stattfand. Die 250 Teilnehmer, die das hervorragend organisierte Symposium besuchten, stammten überwiegend aus Lateinamerika und Spanien. Dieser Tagungsbericht konzentriert sich auf einige Aspekte des Abfallmanagements, die den spezifischen Fall Lateinamerika charakterisieren.

Das Symposium wurde vom „Red de Ingenieria de Saneamiento Ambiental - REDISA“ (Netzwerk zur Erforschung der Verbesserung der Umweltqualität) gemeinsam mit der Universidad del Norte ausgerichtet. REDISA wurde 2003 gegründet und zunächst finanziert von der 
„Agencia Internacional de Cooperación Internacional (AECI)"; seit 2008 kommt die Finanzierung aus dem „Programa Iberoamericano de Ciencia y Tecnología para el Desarrollo (CYTED)". Aufgabe von REDISA ist es, eine Plattform zu schaffen, innerhalb der die Universitäten, die an dem Netzwerk beteiligt sind, Erfahrungen auf dem Gebiet der Erforschung der Umweltqualität und insbesondere dem nachhaltigen Management von Abfällen austauschen sowie ihre Lehre und Forschung auf diesem Gebiet verbessern können. Ursprünglich bestand das Netzwerk aus drei spanischen Universitäten (Universitat Jaume I, Universidad Politécnica de Madrid und der Universidad de Cantabria) und drei lateinamerikanischen Universitäten (Universidad del Norte, Colombia, Pontificia Universidad Católica de Valparaíso, Chile, und der Universidad Nacional de Cuyo, Argentina). 2008 kamen die Universidad Federal de Paraíba (Brasil), die Universidad Autónoma de Baja California, die Universidad Michoacana de San Nicolás de Hidalgo (México) und die Universidad de los Andes (Venezuela) hinzu.

Die Vorträge fanden in drei parallelen Sessions statt. Das Interesse des Autors dieses Berichts an der Konferenz, auf der er selbst auch einen Vortrag hielt, bestand darin, dass er im Rahmen des Projekts „Risk Habitat Megacity“ für die Bearbeitung des Themenfeldes „Abfallmanagement in Santiago de Chile" verantwortlich ist und dabei auch mit der Pontificia Universidad Católica de Valparaíso in Chile zusammenarbeitet. Die Konferenz bot eine hervorragende Möglichkeit, Informationen zum aktuellen Stand der Forschung im Bereich Abfallmanagement in Lateinamerika zu sammeln und sie mit dem europäischen Forschungsstand zu vergleichen. Die Vorträge auf der Konferenz konnten folgenden Themenfeldern zugeordnet werden:

a) „Abfallcharakterisierung“ (Abfälle aus Haushalt bzw. Industrie, Elektronikabfälle, gefährliche Abfälle, Zusammensetzung der Abfälle in ländlichen und städtischen Regionen etc.),

b) „Umweltpolitik und Umweltmanagement",

c) „Behandlung, Verwertung und Entsorgung von Abfällen" (Trennung und Recycling, Deponierung, Kompostierung und Vergärung, energetische Nutzung, Sickerwasserbehandlung auf Deponien etc.), d) „Entsorgung“ (Deponienachsorge, Methanbildung),

e) „Umweltauswirkungen“ (im wesentlichen hervorgerufen durch den Betrieb von Deponien),

f) „soziale Aspekte“ (Information und Fortbildung der Bevölkerung zu den Themen Abfalltrennung und Abfallsammlung, Rolle des informellen Sektors) sowie

g) „Abfallbehandlungstechnologien“.

In dem Vortrag von Salazar Gamez Lorena Lucia (Universidad del Norte in Barranquilla) wurde ein Überblick über das Abfallmanagement in Lateinamerika und in Kolumbien gegeben. Der Anteil der organischen Fraktion in den Siedlungsabfällen ist in den Ländern Lateinamerikas höher als in hoch entwickelten Ländern. Je niedriger das Bruttoinlandsprodukt sei, desto höher sei der organische Anteil im Abfall und desto geringer der wieder verwertbare Anteil an Plastik, Papier und Metallen. In Lateinamerika fallen - so Lucia - täglich ca. 275.000 Tonnen Abfall an. Davon werden 75 Prozent eingesammelt und hiervon wiederum lediglich 30 Prozent auf geordneten Deponien abgelagert. Der Rest gelangt auf ungeordnete Deponien ohne Sickerwasserbehandlung und wird dort teilweise unkontrolliert verbrannt. Diese Deponien liegen z. T. auch in dicht besiedelten Gebieten.

In Kolumbien fallen täglich ca. 27.500 Tonnen an Siedlungsabfällen an (das entspricht ca. 0,65 kg pro Einwohner und Tag; der entsprechende Wert für Deutschland liegt bei ca. $1,6 \mathrm{~kg}$ pro Einwohner und Tag). Hiervon würden 97 Prozent eingesammelt. Die Anzahl der geordneten Deponien nahm in den letzten Jahren ständig zu und damit stieg auch der Anteil an Siedlungsabfällen, der ordnungsgemäß deponiert wurde. Allerdings gebe es immer noch eine Vielzahl von Kommunen, in denen ein großer Anteil des anfallenden Siedlungsabfalls nicht ordnungsgemäß abgelagert wird. Um diese Situation zu verbessern, gebe es jedoch eine Vielzahl von gesetzlichen Regelungen, die eine ungeordnete Deponierung verhindern sollen und die auch den Betrieb und die Nachsorge von Deponien regeln. In den folgenden Passagen werden Ergebnisse zusammengestellt, die den Kontext der Abfallentsorgung in den Ländern Lateinamerikas beschreiben und aus verschiedenen Vorträgen stammen. 
In Lateinamerika wird der überwiegende Anteil des Siedlungsabfalls ohne jegliche Vorbehandlung deponiert. Die Recyclingquote ist relativ gering, man ist aber bestrebt, diese Quote zu erhöhen. Insbesondere in den großen Städten hat der informelle Sektor den größten Anteil an der Recyclingquote. Vereinzelt gibt es kleinere Projekte zur Abtrennung und anschließenden Kompostierung oder Vergärung der organischen Fraktion im Siedlungsabfall. Eine Verbrennung von Siedlungsabfällen in Müllverbrennungsanlagen findet nicht statt.

Bei der Zersetzung der organischen Fraktion der Siedlungsabfälle in Deponien entstehen Deponiegase (im wesentlichen Kohlendioxid und Methan), die zum Treibhauseffekt beitragen. Zur Reduzierung der Freisetzungsmenge können die Deponiegase in Rohrleitungen gefasst und anschließend verbrannt werden. Diese Technologie wird im Rahmen von Projekten im Rahmen der Clean-Development-Mechanism-Maßnahmen finanziell gefördert und wurde daher in den letzten Jahren bereits auf einigen Deponien, so z. B. in Santiago de Chile, realisiert. Eine energetische Nutzung der gesammelten Deponiegase bietet zwar weitere ökologische Vorteile, wird aber bisher nicht umgesetzt, da sie sich i. a. finanziell nicht rentiert. Die einzelnen Vorträge können unter http://www.uninorte.edu.co/residuosredisa/ secciones.asp? $\mathrm{ID}=187$ eingesehen werden.

$\langle\langle\rangle$

\section{Im Fokus: Interdependenzen zwischen Technischem und Kulturellem}

\author{
Einführende Bemerkung zur KIT-Re- \\ levanz der im Folgenden vorgestellten \\ Tagungsthemen
}

\section{von Gerhard Banse, ITAS}

Die wechselseitigen Beziehungen zwischen Technik und Kultur werden in diversen wissenschaftlichen Disziplinen in zunehmendem Maße reflektiert. In der Technikphilosophie und -soziologie, in den Kulturwissenschaften oder in der Interkulturellen Kommunikationswissenschaft sind unterschiedliche Verständnisse von Technik und Kultur zugrunde gelegt, z. B. wird von „Technik und/als Kultur“, von der „Kulturalität des Technischen“, von der „Technizität des Kulturellen“ oder von „Kultürlichkeit der Technik“ gesprochen. Auch wird das Kulturelle im Vergleich etwa zum Sozialen als „ResidualKategorie" verstanden.

Hinter all diesen verschiedenen Ansätzen steht als gemeinsames erkenntnisleitendes Interesse, Zusammenhängen nachzuspüren, die etwa durch globalen Techniktransfer oder Kommunikationsprozesse mit den bisherigen wissenschaftlichen Ansätzen nicht oder nicht ausreichend in den Blick gerieten. Diesem Trend wollen sich das KIT und ITAS nicht verschließen. Im Gegenteil, es wird initiativreich agiert: Im KIT gibt es den Kompetenzbereich „Technik, Kultur und Gesellschaft“, u. a. mit den Kompetenzfeldern „Kulturerbe und sozialer Wandel“" sowie „Wechselwirkung von Wissenschaft, Technik und Gesellschaft". Dieses Kompetenzfeld hat eine Forschergruppe „Technik und Kultur“. Und der KITSchwerpunkt „Mensch und Technik“ beinhaltet auch den Topic „Kultur und Technik“. ITAS ist darin von Anfang an integriert, denn im Helmholtz-Programm ,Technology, Innovation \& Society. Topic 1: Science and Society: Challenges and Expectations“ ist die Untersuchung der ,relation between technologies and their impact on cultural patterns" als Aufgabe festgeschrieben. 